\title{
Entanglement of Grassmannian Coherent States for Multi-Partite $n$-Level Systems
}

\author{
Ghader NAJARBASHI and Yusef MALEKI \\ Department of Physics, University of Mohaghegh Ardabili, Ardabil, 179, Iran \\ E-mail: najarbashi@uma.ac.ir,ymaleki@uma.ac.ir
}

Received September 05, 2010, in final form January 19, 2011; Published online January 24, 2011 doi:10.3842/SIGMA.2011.011

\begin{abstract}
In this paper, we investigate the entanglement of multi-partite Grassmannian coherent states (GCSs) described by Grassmann numbers for $n>2$ degree of nilpotency. Choosing an appropriate weight function, we show that it is possible to construct some wellknown entangled pure states, consisting of GHZ, W, Bell, cluster type and bi-separable states, which are obtained by integrating over tensor product of GCSs. It is shown that for three level systems, the Grassmann creation and annihilation operators $b$ and $b^{\dagger}$ together with $b_{z}$ form a closed deformed algebra, i.e., $S U_{q}(2)$ with $q=e^{\frac{2 \pi i}{3}}$, which is useful to construct entangled qutrit-states. The same argument holds for three level squeezed states. Moreover combining the Grassmann and bosonic coherent states we construct maximal entangled super coherent states.
\end{abstract}

Key words: entanglement; Grassmannian variables; coherent states

2010 Mathematics Subject Classification: 81R30; 15A75; 81P40

\section{Introduction}

Quantum entanglement has been recognized as the vital resource for the applications of quantum information and quantum computation. The emergence of entanglement is a fundamental difference between classical and quantum composite systems. Consequently The question of entanglement of composite systems has been intensively studied in last years $[1,2]$.

In the same direction, the growth of research in theoretical physics and quantum optics has revealed the importance of the coherent states and hence the entanglement of the bosonic, $\mathrm{su}(2)$ and $\mathrm{su}(1,1)$ coherent states has been widely investigated $[3,4,5,6,7,8,9,10,11]$. On the other hand, studying the entanglement properties of Grassmannian coherent states (GCSs) which is defined as a eigenstate of the annihilation operator with Grassmannian eigenvalue [12, 13, 14], remains as a challenging problem of quantum information theory [15, 16, 17, 18]. In [19] we have investigated the relation between entanglement and fermionic coherent states [20].

Aim of the present work is to generalize our previous attempt in [19] to multi-level GCS. Choosing some appropriate weight functions, we show that it is possible to construct some entangled pure states, consisting of GHZ, W, Bell, cluster type and bi-separable states, by tensor product of GCSs. It is shown that for three level systems, the Grassmann creation and annihilation operators $b$ and $b^{\dagger}$ together with $b_{z}$ form a closed deformed algebra, i.e., $S U_{q}(2)$ with $q=e^{\frac{2 \pi i}{3}}$. Based on this algebra and corresponding GCS we construct some entangled qutrit-states. Similar discussion is made for three level Grassmannian squeezed states. Moreover combining the Grassmannian and bosonic coherent states we construct maximal entangled super coherent state.

The paper is organized as follows. In Section 2, the GCS for $n$ level system is introduced. In Section 3, the entangled GCS is studied and explicit examples of multi-qubit entangled states 
such as Bell, W, GHZ, bi-separable and cluster type states are constructed. Moreover, entangled qutrit states based on coherent and squeezed states are presented and then the discussion is generalized for entangled multi-qudit states. Finally combining Grassmannian and bosonic coherent states we construct maximal entangled super coherent state. A brief conclusion is given in Section 4.

\section{Grassmannian coherent states}

Grassmann variables and their applications have been discussed in [12, 20, 21, 22, 23, 24, 25]. Here we review the properties which are particularly appealing for our purposes. Grassmann algebra is generated by variables like $\theta_{i} s$ which have the following properties

$$
\theta_{i} \theta_{j}=q \theta_{j} \theta_{i}, \quad i, j=1,2, \ldots, \quad i<j, \quad \theta_{i}^{n}=0, \quad q=e^{\frac{2 \pi i}{n}} .
$$

Likewise, for the Hermitian conjugate of $\theta, \theta^{\dagger}=\bar{\theta}$, we have

$$
\bar{\theta}_{i} \bar{\theta}_{j}=q \bar{\theta}_{j} \bar{\theta}_{i}, \quad i<j, \quad \bar{\theta}_{i}^{n}=0 .
$$

One has the Berezin's rule of integration as follows

$$
\int d \theta \theta^{k}=\int d \bar{\theta} \bar{\theta}^{k}=\delta_{k, n-1},
$$

where $k$ is a positive integer. Moreover we have the relations

$$
\begin{array}{ll}
\theta d \bar{\theta}=q d \bar{\theta} \theta, & \bar{\theta} d \theta=q d \theta \bar{\theta} \\
\theta d \theta=\bar{q} d \theta \theta, & \bar{\theta} d \bar{\theta}=\bar{q} d \bar{\theta} \bar{\theta} \\
d \theta d \bar{\theta}=\bar{q} d \bar{\theta} d \theta, & \theta \bar{\theta}=\bar{q} \bar{\theta} \theta
\end{array}
$$

The quantization relations between number states $\{|m\rangle, m=0,1,2, \ldots, n-1\}$ and Grassmann variables $\theta, \bar{\theta}$ are

$$
\theta|m\rangle=q^{m-1}|m\rangle \theta, \quad \theta\langle m|=\bar{q}^{m-1}\langle m| \theta .
$$

By definition, GCS denoted by $|\theta\rangle_{n}$, is the eigenstate of annihilation operator $b$ with eigenvalue $\theta$ that is

$$
b|\theta\rangle_{n}=\theta|\theta\rangle_{n}
$$

where $\theta$ is a complex Grassmann variable with the following relations

$$
\left[\theta, b^{\dagger}\right]_{q}=[b, \theta]_{q}=0,
$$

where $[A, B]_{q}:=A B-q B A$. One may tempt to define the annihilation operator $b$ as

$$
b=\sum_{m=0}^{n-1} \sqrt{m+1}|m\rangle\langle m+1| .
$$

Hence $|\theta\rangle_{n}$ can be define as below

$$
|\theta\rangle_{n}=\sum_{m=0}^{n-1} \frac{\bar{q}^{\frac{m(m+1)}{2}}}{\sqrt{m !}} \theta^{m}|m\rangle=: e^{\left(b^{\dagger} \theta\right)}|0\rangle .
$$

One may regard $D(\theta):=e^{\left(b^{\dagger} \theta\right)}$ as the displacement operator for GCS. 


\section{Entangled Grassmann coherent states}

Consider a linear combination of tensor product of Grassmannian coherent states as follows

$$
|\psi\rangle=\sum_{i_{1}, i_{2}, \ldots, i_{n}} f_{i_{1}, i_{2}, \ldots, i_{n}}\left|\theta_{i_{1}}\right\rangle\left|\theta_{i_{2}}\right\rangle \cdots\left|\theta_{i_{n}}\right\rangle .
$$

Some times, if we take a proper form of the tensor product of Grassmannian coherent states, it is possible to find an appropriate weight function to make the right hand side of the following equation to be a maximally entangled state like Bell, cluster type, GHZ and $\mathbf{W}$ states, i.e.,

$$
\int d \theta_{i_{1}} d \theta_{i_{2}} \cdots d \theta_{i_{n}} w\left(\theta_{i_{1}}, \ldots, \theta_{i_{n}}\right)|\psi\rangle=|\gamma\rangle,
$$

where $w\left(\theta_{i_{1}}, \ldots, \theta_{i_{n}}\right)$ is a proper weight function, and the state $|\gamma\rangle$, is maximally entangled state. We note that the weight functions is not unique and of course for a given state there may not be such a function at all. Here there is no need to normalize GCSs since it can be included in the weight functions.

\subsection{Multi-qubit states}

Two level Grassmannian coherent state can be written as

$$
|\theta\rangle=|0\rangle-\theta|1\rangle
$$

At first, we consider simple cases that yield maximally entangled Bell states as follows

$$
\begin{aligned}
& \int d \theta\left(\frac{\mp 1}{2 \sqrt{2}}\right)[|\theta\rangle| \pm \theta\rangle-|-\theta\rangle|\mp \theta\rangle]=\frac{1}{\sqrt{2}}(|01\rangle \pm|10\rangle)=\left|\Psi^{ \pm}\right\rangle, \\
& \int d \bar{\theta} d \theta\left(\frac{ \pm 1}{\sqrt{2}} e^{ \pm \theta \bar{\theta}}\right)|\bar{\theta}\rangle|\theta\rangle=\frac{1}{\sqrt{2}}(|00\rangle \pm|11\rangle)=\left|\Phi^{ \pm}\right\rangle .
\end{aligned}
$$

One gets the general form of the $\mathbf{W}$ state as follows

$$
\int d \theta\left(\frac{-1}{\sqrt{n}}\right) \underbrace{|\theta\rangle|\theta\rangle \cdots|\theta\rangle}_{n \text { times }}=\frac{1}{\sqrt{n}}(|100 \ldots 0\rangle+|010 \ldots 0\rangle+\cdots+|0 \ldots 001\rangle)=\left|\mathbf{W}^{(n)}\right\rangle .
$$

Likewise, we can construct the general form of GHZ state as follows

$$
\int d \theta_{1} d \theta_{2} \cdots d \theta_{n} w\left|\theta_{n}\right\rangle\left|\theta_{n-1}\right\rangle \cdots\left|\theta_{1}\right\rangle=\frac{1}{\sqrt{2}}(|00 \ldots 0\rangle+|11 \ldots 1\rangle)=\left|\mathbf{G H Z}^{(n)}\right\rangle,
$$

where

$$
w=\frac{1}{\sqrt{2}}\left((-1)^{\left[\frac{n}{2}\right]}+\theta_{n} \theta_{n-1} \cdots \theta_{1}\right) .
$$

Note that unlike $\mathbf{W}^{(n)}$, we can not construct $\mathbf{G} \mathbf{H Z} \mathbf{Z}^{(n)}$ using just one Grassmann variable for $n>2$. The amount of entanglement of these states can be evaluated using the purity which is defined as $p(|\psi\rangle)=\frac{2}{n} \sum_{i} \operatorname{tr} \rho_{i}^{2}-1$, where $\rho_{i}$ is reduced density matrix of qubit $i$ [26]. For $\mathbf{G H Z}^{(n)}$ state the purity is zero and for $\mathbf{W}^{(n)}$ state reads $\left(\frac{n-2}{n}\right)^{2}$, which implies that $\mathbf{G H Z} \mathbf{H}^{(n)}$ state is maximally entangled while in the limit $n \rightarrow \infty$ the purity goes to 1 for $\mathbf{W}^{(n)}$ hence it become separable. The other set of important entangled states are cluster type states [27]. Here we consider one example of these states as follows

$$
\left|\operatorname{CLUSTER}^{ \pm}\right\rangle=\frac{1}{2}( \pm|0000\rangle+|0011\rangle+|1100\rangle \mp|1111\rangle) .
$$


If we take $|\psi\rangle=\left|\theta_{1}\right\rangle\left|\theta_{2}\right\rangle\left|\theta_{3}\right\rangle\left|\theta_{4}\right\rangle$, in equation (2), then we get

$$
\int d \theta_{1} \cdots d \theta_{4} w_{\left(\mathrm{CLUSTER}^{ \pm}\right)}\left|\theta_{1}\right\rangle\left|\theta_{2}\right\rangle\left|\theta_{3}\right\rangle\left|\theta_{4}\right\rangle=\left|\mathrm{CLUSTER}^{ \pm}\right\rangle
$$

where

$$
w_{\left(\text {CLUSTER }^{ \pm}\right)}=\frac{1}{2}\left( \pm \theta_{4} \theta_{3} \theta_{2} \theta_{1}+\theta_{2} \theta_{1}+\theta_{4} \theta_{3} \mp 1\right) .
$$

Taking the same method, one can construct the other cluster type states with appropriate weight functions.

\subsection{Multi-qutrit states and $S U_{q}(2)$ deformed algebra}

Multi-qutrit states are three level systems. Therefore, we take the following bases to describe these states

$$
|0\rangle \equiv\left(\begin{array}{c}
1 \\
0 \\
0
\end{array}\right), \quad|1\rangle \equiv\left(\begin{array}{l}
0 \\
1 \\
0
\end{array}\right), \quad|2\rangle \equiv\left(\begin{array}{l}
0 \\
0 \\
1
\end{array}\right)
$$

The associated Grassmann numbers satisfy $\theta^{3}=0$. So, the following quantization relations hold

$$
\begin{aligned}
\theta|0\rangle & =\bar{q}|0\rangle \theta, & & \langle 0| \bar{\theta}=q \bar{\theta}\langle 0|, \\
\theta|1\rangle & =|1\rangle \theta, & & \langle 1| \bar{\theta}=\bar{\theta}\langle 1|, \\
\theta|2\rangle & =q|2\rangle \theta, & & \langle 2| \bar{\theta}=\bar{q} \bar{\theta}\langle 2|,
\end{aligned}
$$

where $q=e^{\frac{2 \pi i}{3}}$. Now, we are going to derive an algebra under which three level GCSs can be constructed. For this purpose, consider the explicit form of the three level annihilation and creation operators $b, b^{\dagger}$ and $b_{z}$ as follows

$$
b:=|0\rangle\langle 1|+\sqrt{2}| 1\rangle\left\langle 2\left|, \quad b^{\dagger}:=\right| 1\right\rangle\langle 0|+\sqrt{2}| 2\rangle\langle 1|, \quad b_{z}:=\left[b, b^{\dagger}\right]_{q}:=b b^{\dagger}-q b^{\dagger} b .
$$

Using the commutation relation between operators $b$ and $b_{z}$, we get

$$
\left[b_{z}, b\right]_{q}=\left(1-2 q+q^{2}\right)|0\rangle\left\langle 1\left|+\left(2-q+2 q^{2}\right) \sqrt{2}\right| 1\right\rangle\langle 2| .
$$

If the right hand side of the above equation is taken to be proportional to $b$, we must have

$$
\left(1-2 q+q^{2}\right)=\left(2-q+2 q^{2}\right)
$$

or equivalently

$$
\left(1+q+q^{2}\right)=0 \Rightarrow q=e^{\frac{2 \pi i}{3}} .
$$

Thus the equation (3) reduces to

$$
\left[b_{z}, b\right]_{q}=-3 q b
$$

Developing the same method to the commutator of $b^{\dagger}$ and $b_{z}$ the condition (4) is obtained again. Hence we have

$$
\left[b, b^{\dagger}\right]_{q}=b_{z}, \quad\left[b_{z}, b\right]_{q}=-3 q b, \quad\left[b^{\dagger}, b_{z}\right]_{q}=-3 q b^{\dagger} .
$$


As we see this algebra is closed and it is reminiscent of the $S U(2)$ algebra, and we call it deformed $S U_{q}(2)$ algebra. The coherent state for three level system is

$$
|\theta\rangle=|0\rangle+\bar{q} \theta|1\rangle+\frac{1}{\sqrt{2}} \theta^{2}|2\rangle=\left(1+b^{\dagger} \theta+\frac{1}{2} \theta^{2} b^{\dagger^{2}}\right)|0\rangle .
$$

Using the second equality we rewrite three level GCS in terms of the exponential function, defined in equation (1) as follows

$$
|\theta\rangle=e^{\left(b^{\dagger} \theta\right)}|0\rangle
$$

Now we will use this coherent state to construct some entangled qutrit states. To do so, we start with generalized Bell states for three level system [28]. These states are

$$
\left|\psi_{ \pm}\right\rangle=\frac{1}{\sqrt{3}}(|00\rangle \pm|11\rangle+|22\rangle), \quad\left|\varphi_{ \pm}\right\rangle=\frac{1}{\sqrt{3}}(|02\rangle \pm|11\rangle+|20\rangle)
$$

In order to obtain the above states, we take the state $\left|\theta_{1}\right\rangle\left|\theta_{2}\right\rangle$. Therefore we have

$$
\int d \theta_{1} d \theta_{2} \frac{1}{\sqrt{3}}\left(\theta_{2}^{2} \theta_{1}^{2} \pm q^{2} \theta_{1} \theta_{2}+2 q\right)\left|\theta_{1}\right\rangle\left|\theta_{2}\right\rangle=\left|\psi_{ \pm}\right\rangle
$$

likewise

$$
\int d \theta_{1} d \theta_{2} \frac{1}{\sqrt{3}}\left(\sqrt{2} \theta_{1}^{2} \pm q^{2} \theta_{1} \theta_{2}+\sqrt{2} \theta_{2}^{2}\right)\left|\theta_{1}\right\rangle\left|\theta_{2}\right\rangle=\left|\varphi_{ \pm}\right\rangle .
$$

Of course the weight function may be chosen in a way that it yields contraction, this means that we may have MES in the subspaces. The following examples are of this type

$$
\begin{aligned}
& \int d \theta_{1} d \theta_{2} \frac{1}{\sqrt{2}}\left(\theta_{2}^{2} \theta_{1}^{2} \pm 2 q\right)\left|\theta_{1}\right\rangle\left|\theta_{2}\right\rangle=\frac{1}{\sqrt{2}}(|00\rangle \pm|22\rangle), \\
& \int d \theta_{1} d \theta_{2} \frac{1}{\sqrt{2}}\left(\theta_{2}^{2} \theta_{1}^{2} \pm q^{2} \theta_{1} \theta_{2}\right)\left|\theta_{1}\right\rangle\left|\theta_{2}\right\rangle=\frac{1}{\sqrt{2}}(|00\rangle \pm|11\rangle),
\end{aligned}
$$

which is comparable with hole burning of an atomic coherent state prepared for a collection of $N$ two-level atoms [29]. One may construct bi-separable states for three partite systems, for instance

$$
\int d \theta_{1} d \theta_{2} d \theta_{3} \frac{1}{\sqrt{3}}\left(\theta_{3}^{2} \theta_{2}^{2} \theta_{1}^{2} \pm \bar{q} \theta_{1}^{2} \theta_{2} \theta_{3}\right)\left|\theta_{1}\right\rangle\left|\theta_{2}\right\rangle\left|\theta_{3}\right\rangle=|0\rangle \otimes \frac{1}{\sqrt{2}}(|00\rangle \pm|11\rangle) .
$$

By changing the weight functions, one may get some other entangled states as well. For example, we can create all MESs introduced in [30]. Here we give an example as follows

$$
\int d \theta_{1} d \theta_{2} \frac{1}{\sqrt{3}}\left(q^{2} \theta_{1}^{2} \theta_{2}+\sqrt{2} \omega \theta_{1}+\sqrt{2} \omega^{2} \theta_{2}^{2}\right)\left|\theta_{1}\right\rangle\left|\theta_{2}\right\rangle=\left|\Psi_{2}^{(2)}\right\rangle
$$

where $\omega^{3}=1$ and

$$
\left|\Psi_{2}^{(2)}\right\rangle=\frac{1}{\sqrt{3}}\left(|01\rangle+\omega|12\rangle+\omega^{2}|20\rangle\right) .
$$




\subsection{Grassmannian qutrit squeezed states}

For three level system, it is possible to define squeezing operator associated to Grassmann number as follows

$$
S(\xi)=\exp \left[\frac{1}{2}\left(\xi b^{\dagger^{2}}-\bar{\xi} b^{2}\right)\right] .
$$

Here instead of $\theta$ we use $\xi$, for three level squeezed state. Noting that in the three level system, the operators $b^{3}$ and $b^{\dagger^{3}}$ vanish and hence the expansion of squeezing operator becomes

$$
S(\xi)=I+\frac{1}{2}\left(\xi b^{\dagger^{2}}-\bar{\xi} b^{2}\right)-\frac{\bar{q}}{8} \xi \bar{\xi}\left(b^{\dagger^{2}} b^{2}+q b^{2} b^{\dagger^{2}}\right) .
$$

It is remarkable that the operators $b^{2}$ and $b^{\dagger^{2}}$, used in squeezing operator for three level system, together with $b_{z}^{\prime}$ obey a closed algebra as follows

$$
\left[b^{\dagger^{2}}, b^{2}\right]:=b_{z}^{\prime}, \quad\left[b_{z}^{\prime}, b^{2}\right]=-8 b^{2}, \quad\left[b_{z}^{\prime}, b^{\dagger^{2}}\right]=8 b^{\dagger^{2}} .
$$

Grassmannian squeezed states by definition can be obtained by applying squeezing operator $S(\xi)$ on the vacuum state $|0\rangle$, i.e.

$$
|\xi\rangle=S(\xi)|0\rangle \text {. }
$$

Therefore for three level system we get the squeezed state as

$$
|\xi\rangle=|0\rangle+\frac{1}{\sqrt{2}} \xi|2\rangle-\frac{1}{4} \xi \bar{\xi}|0\rangle=\left(1-\frac{1}{4} \xi \bar{\xi}\right)|0\rangle+\frac{1}{\sqrt{2}} \xi|2\rangle
$$

Therefore we can construct entangled state, $\frac{1}{\sqrt{2}}(|00\rangle+|22\rangle)$ just by one Grassmannian squeezed state $|\xi\rangle$ which such construction is not possible by GCS with one Grassman number, i.e.,

$$
\int d \bar{\xi} d \xi \frac{1}{\sqrt{2}}\left(2 \bar{q} \bar{\xi}^{2}-16 \bar{q}-2 \bar{q} \xi \bar{\xi}+\bar{\xi}^{2} \xi^{2}\right)|\xi\rangle|\xi\rangle=\frac{1}{\sqrt{2}}(|00\rangle+|22\rangle) .
$$

Now, we construct entangled state using tensor product of the coherent and squeezed states as follows

$$
\int d \theta(q+\theta) \widetilde{|\theta\rangle}|\theta\rangle=\frac{1}{\sqrt{2}}(|02\rangle+|20\rangle),
$$

where $\widetilde{|\theta\rangle}$ is squeezed state (6), (which $\xi$ is replace with $\theta$ ) and $|\theta\rangle$ is GCS for three level system. It is notable that, in this case we have used one Grassmann number and obtained the state $\frac{1}{\sqrt{2}}(|02\rangle+|20\rangle)$, which is not possible to be obtained using tensor product of GCSs with just one Grassmann number, i.e., there is no weight function $w$ such that $\int d \theta w|\theta\rangle|\theta\rangle=\frac{1}{\sqrt{2}}(|02\rangle+|20\rangle)$.

\subsection{Multi-qudit entangled states}

We should mention that it is easy to generalize this method to any $Z_{n}$ graded GCS. To this aim let us start with the general form of the GCS

$$
|\theta\rangle_{n}=\sum_{m=0}^{n-1} \frac{\bar{q}^{\frac{m(m+1)}{2}}}{\sqrt{m !}} \theta^{m}|m\rangle,
$$


we product two GCSs with different Grassmann numbers as

$$
\left|\theta_{1}\right\rangle_{n}\left|\theta_{2}\right\rangle_{n}=\sum_{i, j=0}^{n-1} c_{i j} \theta_{1}^{i} \theta_{2}^{j}|i\rangle|j\rangle, \quad \text { where } \quad c_{i j}=\frac{q^{\frac{(j-i)-(i+j)^{2}}{2}}}{\sqrt{i ! j !}} .
$$

In [31] it has been shown that the symmetric states are either globally entangled or fully separable with all the constituent systems having identical states, whereas antisymmetric states are globally entangled. By globally entangled we mean that the state remains entangled across any bi-partition. On the other hand a state is fully separable if it remains separable across all bi-partitions. Therefore we may determine the weight function in a way that the obtained state becomes symmetric MES after integration, i.e.,

$$
\begin{aligned}
& \int d \theta_{1} d \theta_{2} w\left|\theta_{1}\right\rangle_{n}\left|\theta_{2}\right\rangle_{n}=\frac{1}{\sqrt{n}} \sum_{i=0}^{n-1}|i\rangle|i\rangle, \\
& \int d \theta_{1} d \theta_{2} w \sum_{i, j=0}^{n-1} c_{i j} \theta_{1}^{i} \theta_{2}^{j}|i\rangle|j\rangle=\frac{1}{\sqrt{n}} \sum_{i=0}^{n-1}|i\rangle|i\rangle .
\end{aligned}
$$

Now consider the general form of Grassmannian weight function

$$
w=\sum_{k, l=0}^{n-1} w_{k, l} \theta_{1}^{k} \theta_{2}^{l}
$$

Putting this weight in equation (9) and taking into account the quantization and the integration rules of generalized Grassmannian variables we have

$$
\begin{aligned}
& \sum_{k, l=0}^{n-1} \sum_{i, j=0}^{n-1} c_{i j} w_{k, l} \int d \theta_{1} d \theta_{2} \theta_{1}^{k} \theta_{2}^{l} \theta_{1}^{i} \theta_{2}^{j}|i\rangle|j\rangle=\frac{1}{\sqrt{n}} \sum_{i=0}^{n-1}|i\rangle|i\rangle, \\
& \sum_{k, l=0}^{n-1} \sum_{i, j=0}^{n-1} c_{i j} w_{k, l} q^{k l+j k+i j} \int d \theta_{1} d \theta_{2} \theta_{2}^{l+j} \theta_{1}^{i+k}|i\rangle|j\rangle=\frac{1}{\sqrt{n}} \sum_{i=0}^{n-1}|i\rangle|i\rangle, \\
& \sum_{k, l=0}^{n-1} \sum_{i, j=0}^{n-1} c_{i j} w_{k, l} q^{k l+j k+i j} \delta_{n-1}^{l+j} \delta_{n-1}^{i+k}|i\rangle|j\rangle=\frac{1}{\sqrt{n}} \sum_{i=0}^{n-1}|i\rangle|i\rangle,
\end{aligned}
$$

where the symbol $\delta_{j}^{i}$ is the usual Kronecker delta. We note that

$$
\delta_{n-1}^{l+j} \delta_{n-1}^{i+k} \neq 0 \quad \Longrightarrow \quad l+j=n-1=i+k .
$$

The equation (10) gives the right hand side MES if the terms with $i \neq j$ vanish, which due to the equation (11) implies that $w_{k, l}=0$ for $k \neq l$. With this explanation the equation (10) reduces to

$$
\sum_{i=0}^{n-1} c_{i i} w_{n-1-i, n-1-i} q^{(n-1-i)(n-1)+i^{2}}|i\rangle|i\rangle=\frac{1}{\sqrt{n}} \sum_{i=0}^{n-1}|i\rangle|i\rangle .
$$

Thus

$$
w=\frac{1}{\sqrt{n}} \sum_{k=0}^{n-1} c_{(n-1-k),(n-1-k)}^{-1} \bar{q}^{k(n-1)+(n-1-k)^{2}} \theta_{1}^{n-1-k} \theta_{2}^{n-1-k} .
$$

With this weight the equation (8) holds. 
We note that any attempt for finding finite deformed $S U_{q}(2)$ algebra, as for qutrit case, for more than three level systems will cease to exist. Actually these generators do not form finite closed algebra at all. In the previous subsection we defined squeezed state for three level system by action the squeezing operator (5) on vacuum state $|0\rangle$. One may tempt to define the squeezed state for general qudit states. Here is a point. Instead of using squeezing operator (5), we use $S(\xi)=e^{\left(\xi b^{\dagger^{2}}\right)}$ to define squeezed state

$$
|\xi\rangle=e^{\left(\xi b^{\dagger^{2}}\right)}|0\rangle
$$

This makes the coefficients change in $|\xi\rangle$. For instance if we take $S(\xi)=e^{\left(\xi b^{\dagger^{2}}\right)}$, then its associated qutrit squeezed state becomes $|0\rangle+\xi|2\rangle$, which up to the coefficients is the equation (6). But the construction of maximally entangled qutrit states go to the same state in (7) no matter how we take the squeezing operator here, i.e.,

$$
\int d \xi \frac{1}{\sqrt{2}}\left(1+\xi^{2}\right)|\xi\rangle|\xi\rangle=\frac{1}{\sqrt{2}}(|00\rangle+|22\rangle) \text {. }
$$

As was clearly seen in qutrit case, the squeezed state is superposition of even number states $|0\rangle$ and $|2\rangle$. This is the case in general. Now we use this operator to develop equation (5) to qudit case. The general squeezed state is

$$
|\xi\rangle=e^{\left(\xi b^{b^{2}}\right)}|0\rangle=\sum_{i=0}^{n-1} \frac{\bar{q}^{i(i-1)}}{i !} \xi^{i}|2 i\rangle .
$$

Thus considering the product of two squeezed states

$$
|\xi\rangle|\xi\rangle=\sum_{i, j=0}^{n-1} d_{i, j} \xi^{i+j}|2 i\rangle|2 j\rangle,
$$

with $d_{i, j}=\frac{\bar{q}^{i(i-1)+j(j-1)+(2 j-1) j}}{i ! j !}$, one can create the maximally entangled state as follows

$$
\int d \theta w|\xi\rangle|\xi\rangle=\frac{1}{\sqrt{n}} \sum_{k=0}^{n-1}|2 k\rangle|2 k\rangle,
$$

where the general form of the one-variable weight function is

$$
w=\sum_{m=0}^{n-1} w_{m} \xi^{m} .
$$

Similar to the $n$ level qudit coherent states we can find the weight function as

$$
w=\frac{1}{\sqrt{n}} \sum_{i=0}^{n-1} \frac{1}{d_{i i}} \xi^{n-2 i-1} .
$$

There is rather tight relation between the construction of entangled states of this work and [32] where they used the same method to characterize the entanglement by polynomials of fermionic nilpotent raising operators $\sigma^{+}$, acting on a reference vacuum state. The difference is that we use Grassmann anticommuting variables instead of Clifford nilpotent variables. For example in two qubit case one may take

$$
F\left(\sigma_{1}^{+}, \sigma_{2}^{+}\right)=a_{0}+a_{1} \sigma_{1}^{+}+a_{2} \sigma_{2}^{+}+a_{3} \sigma_{1}^{+} \sigma_{2}^{+},
$$

which acting on vacuum state $|00\rangle$ yields, $a_{0}|00\rangle+a_{1}|10\rangle+a_{2}|01\rangle+a_{3}|11\rangle$. So one can take the coefficients such that the state becomes MES. As a simple example taking $a_{0}=a_{3}=\frac{1}{\sqrt{2}}$ and $a_{1}=a_{2}=0$ yields Bell state $\left|\Phi^{+}\right\rangle$. 


\subsection{Entangled supper coherent state}

The study of entanglement in a system involve both bosons and fermions remains one of the most challenging problems in quantum information science [15, 17]. Here we want to show that in some cases it is possible to construct MES using superposition of bosonic and fermionic coherent states. Fermions are described in the anti-commuting Grassmann coordinate space, consequently one must use super-Hilbert space to study both bosons and fermions. A bosonic coherent state can be defined as eigenstate of the annihilation operator

$$
b|\alpha\rangle=\alpha|\alpha\rangle,
$$

where $\alpha$ is a complex number, and $b$ is annihilation operator for the bosonic coherent state

$$
|\alpha\rangle=e^{\frac{-|\alpha|^{2}}{2}} \sum_{n=0}^{\infty} \frac{\alpha^{n}}{\sqrt{n !}}|n\rangle=D(\alpha)|0\rangle,
$$

and $D(\alpha)$, is displacement operator

$$
D(\alpha):=\exp \left(a^{\dagger} \alpha-\alpha^{*} a\right) .
$$

We can also express that

$$
\langle\alpha \mid \beta\rangle=e^{-\frac{1}{2}\left(|\alpha|^{2}+|\beta|^{2}-2 \alpha^{*} \beta\right)} .
$$

With this background, let $|\alpha\rangle$ and $|\beta\rangle$ be two orthogonal bosonic coherent states, which is possible in the limit $\alpha \rightarrow \infty$ and $\beta \rightarrow 0$. Therefore in the space spanned by two coherent states one can take $|\alpha\rangle \equiv|1\rangle_{b}$ and $|\beta\rangle \equiv|0\rangle_{b}$. On the other hand in the space of fermionic system we can describe the states $|1\rangle_{f}$ and $|0\rangle_{f}$ integrating on fermionic coherent states. Now consider the following super coherent states belonging to the Hilbert space $\mathcal{H}_{\text {fermion }} \otimes \mathcal{H}_{\text {boson }}$

$$
\lim _{\alpha \rightarrow \infty} \int d \theta \frac{\theta}{\sqrt{2}}|\theta\rangle|\alpha\rangle \mp \lim _{\beta \rightarrow 0} \int d \theta \frac{1}{\sqrt{2}}|\theta\rangle|\beta\rangle=\frac{1}{\sqrt{2}}\left(|0\rangle_{f}|1\rangle_{b} \pm|1\rangle_{f}|0\rangle_{b}\right),
$$

and likewise

$$
\lim _{\alpha \rightarrow 0} \int d \theta\left(\frac{-1}{\sqrt{2}}\right)|\theta\rangle|\alpha\rangle \pm \lim _{\beta \rightarrow \infty} \int d \theta \frac{\theta}{\sqrt{2}}|\theta\rangle|\beta\rangle=\frac{1}{\sqrt{2}}\left(|0\rangle_{f}|0\rangle_{b} \pm|1\rangle_{f}|1\rangle_{b}\right) .
$$

The right hand sides of the above equations may be interpreted as two partite MESs in the Hilbert space $\mathcal{H}_{\text {fermion }} \otimes \mathcal{H}_{\text {boson }}$. We not that, if $\alpha \rightarrow \beta$, then we obtain separable states, i.e.,

$$
\lim _{\alpha \rightarrow \beta} \int d \theta \frac{\theta}{\sqrt{2}}|\theta\rangle|\alpha\rangle \mp \int d \theta \frac{1}{\sqrt{2}}|\theta\rangle|\beta\rangle=\frac{1}{\sqrt{2}}\left(|0\rangle_{f} \pm|1\rangle_{f}\right)|\alpha\rangle .
$$

Of course the other way to create the orthogonal basis in the bosonic Hilbert space is

$$
|0\rangle_{b}=|\alpha\rangle, \quad|1\rangle_{b}=\frac{|\beta\rangle-\langle\alpha \mid \beta\rangle|\alpha\rangle}{N_{1}}, \quad \text { where } \quad N_{1}=\sqrt{1-|\langle\alpha \mid \beta\rangle|} .
$$

Thus instead of taking limit we can use the above bases to construct MESs in $\mathcal{H}_{\text {fermion }} \otimes \mathcal{H}_{\text {boson }}$, however in spite of the fact that it belongs to $\mathcal{H}_{\text {boson }}|1\rangle_{b}$ is not a coherent state anymore.

The approach used here is somewhat different from the method used by Castellani et al. in [17]. They established the superqubits states in composed Hilbert space of the form $H_{\text {boson }} \oplus$ $H_{\text {fermion, }}$ with general form

$$
|\psi\rangle=\sum_{i}^{n} b_{i}\left|B_{i}\right\rangle+\theta \sum_{i}^{n} f_{i}\left|F_{i}\right\rangle,
$$


with $n$ bosonic states $\left|B_{i}\right\rangle$ and $n$ fermionic states $\left|F_{i}\right\rangle$, combined via a single Grassmann coordinate $\theta$ and then normalized it with suitable weight function. For identical particles they symmetrized tensor product superqubits to produce the entangled states such as

$$
|\psi\rangle \otimes\left|\psi^{\prime}\right\rangle=\sum_{i, j}^{n} b_{i} b_{j}^{\prime}\left|B_{i} B_{j}\right\rangle+\theta \sum_{i, j}^{n}\left(b_{i} f_{j}^{\prime}+b_{i}^{\prime} f_{j}\right)\left|B_{i} F_{j}\right\rangle .
$$

Basically there are some differences between these constructions. For example, we begin to establish fermionic state as an eigenstate of the fermionic annihilation operator, while the above superqubit state has no connection to such operator at all. On the other hand there is no need to normalize GCSs beforehand since the normalization factor can be included in the weight function.

\section{Conclusion}

In conclusion, we have investigated the entanglement of multi-partite Grassmannian coherent states (GCS) described by anti-commuting Grassmann numbers. This task is achieved by integration over Grassmann numbers with taking appropriate weight functions. In other words we established a relation between GCS and pure entangled states which are treated almost separately. The construction of GHZ, W, Bell, cluster type and bi-separable states and also generalization of entanglement for $n$ level GCSs was developed. It is shown that for three level systems, the creation and annihilation operators $b$ and $b^{\dagger}$ together with $b_{z}:=\left[b, b^{\dagger}\right]_{q}$ form a closed deformed algebra $S U_{q}(2)$. The similar closed algebra was obtained for three operators $b^{2}, b^{\dagger^{2}}$ and $b_{z}^{\prime}:=\left[b^{2}, b^{t^{2}}\right]$, which one may tempt to define Grassmannian squeezed state and create some MESs using these squeezed states. For three level systems, combination of Grassmannian coherent and squeezed states yields other MESs which was not possible to be obtained using GCSs with just one Grassmann number before.

Finally combining the Grassmann and bosonic coherent states we construct maximal entangled super coherent states.

\section{References}

[1] Nielsen M.A., Chuang I.L., Quantum computation and quantum information, Cambridge University Press, Cambridge, 2000.

[2] Petz D., Quantum information theory and quantum statistics, Springer-Verlag, Berlin, 2008.

[3] van Enk S.J., Decoherence of multidimensional entangled coherent states, Phys. Rev. A 72 (2005), 022308, 6 pages, quant-ph/0503207.

[4] van Enk S.J., Hirota O., Entangled coherent states: teleportation and decoherence, Phys. Rev. A 64 (2001), 022313, 6 pages, quant-ph/0012086.

[5] Fujii K., Introduction to coherent states and quantum information theory, quant-ph/0112090.

[6] Najarbashi G., Maleki Y., Maximal entanglement of two-qubit states constructed by linearly independent coherent states, arXiv:1007.1387.

[7] Fu H., Wang X., Solomon A.I., Maximal entanglement of nonorthogonal states: classification, Phys. Lett. A 291 (2001), 73-76, quant-ph/0105099.

[8] Wang X., Sanders B.C., Multipartite entangled coherent states, Phys. Rev. A 65 (2001), 012303, 7 pages, quant-ph/0104011.

[9] Wang X., Bipartite entangled non-orthogonal states, J. Phys. A: Math. Gen. 35 (2002), 165-173, quant-ph/0102011.

[10] Wang X., Sanders B.C., Pan S.-H., Entangled coherent states for systems with $S U(2)$ and $S U(1,1)$ symmetries, J. Phys. A: Math. Gen. 33 (2000), 7451-7467, quant-ph/0001073. 
[11] Wang X., Quantum teleportation of entangled coherent states, Phys. Rev. A 64 (2001), 022302, 4 pages, quant-ph/0102048.

[12] Majid S., Rodríguez-Plaza M.J., Random walk and the heat equation on superspace and anyspace, J. Math. Phys. 35 (1994), 3753-3760.

[13] Cabra D.C., Moreno E.F., Tanasa A., Para-Grassmann variables and coherent states, SIGMA 2 (2006), 087, 8 pages, hep-th/0609217.

[14] Najarbashi G., Fasihi M.A., Fakhri H., Generalized Grassmannian coherent states for pseudo-Hermitian n-level systems, J. Phys. A: Math. Theor. 43 (2010), 325301, 10 pages, arXiv:1007.1392.

[15] Borsten L., Dahanayake D., Duff M.J., Rubens W., Superqubits, Phys. Rev. D 81 (2010), 105023, 16 pages, arXiv:0908.0706.

[16] Khanna F.C., Malbouisson J.M.C., Santana A.E., Santos E.S., Maximum entanglement in squeezed boson and fermion states, Phys. Rev. A 76 (2007), 022109, 5 pages, arXiv:0709.0716.

[17] Castellani L., Grassi P A., Sommovigo L., Quantum computing with superqubits, arXiv:1001.3753.

[18] Najarbashi G., Fasihi M.A., Mirmasoudi F., Mirzaei S., Entanglement of fermionic coherent states for pseudo Hermitian Hamiltonian, Poster at International Iran Conference on Quantum Information-2010 (2010, Kish Island, Iran).

[19] Najarbashi G., Maleki Y., Entanglement in multi-qubit pure fermionic coherent states, arXiv:1004.3703.

[20] Cahill K.E., Glauber R.J., Density operators for fermions, Phys. Rev. A 59 (1999), 1538-1555, physics/9808029.

[21] Kerner R., $Z_{3}$-graded algebras and the cubic root of the supersymmetry translations, J. Math. Phys. 33 (1992), 403-411.

[22] Filippov A.T., Isaev A.P., Kurdikov A.B., Para-Grassmann differential calculus, Theoret. and Math. Phys. 94 (1993), 150-165, hep-th/9210075.

[23] Isaev A.P., Para-Grassmann integral, discrete systems and quantum groups, Internat. J. Modern Phys. A 12 (1997), 201-206, q-alg/9609030.

[24] Cugliandolo L.F., Lozano G.S., Moreno E.F., Schaposnik F.A., A note on Gaussian integrals over paraGrassmann variables, Internat. J. Modern Phys. A 19 (2004), 1705-1714, hep-th/0209172.

[25] Ilinski K.N., Kalinin G.V., Stepanenko A.S., q-functional Wick's theorems for particles with exotic statistics, J. Phys. A: Math. Gen. 30 (1997), 5299-5310, hep-th/9704181.

[26] Barnum H., Knill E., Ortiz G., Somma R., Viola L., A subsystem-independent generalization of entanglement, Phys. Rev. Lett. 92 (2004), 107902, 4 pages, quant-ph/0305023.

[27] Munhoz P.P., Semião F.L., Vidiella-Barranco A., Cluster-type entangled coherent states, Phys. Lett. A 372 (2008), 3580-3585, arXiv:0705.1549.

[28] Fujii K., A relation between coherent states and generalized Bell states, quant-ph/0105077.

[29] Gerry C.C., Peart M., Spin squeezing and entanglement via hole-burning in atomic coherent states, Phys. Lett. A 372 (2008), 6480-6483.

[30] Sun C., Xue K., Wang G., Wu C., A study on the relations between the topological parameter and entanglement, arXiv:1001.4587.

[31] Ichikawa T., Sasaki T., Tsutsui I., Yonezawa N., Exchange symmetry and multipartite entanglement, Phys. Rev. A 78 (2008), 052105, 8 pages, arXiv:0805.3625.

[32] Mandilara A., Akulin V.M., Smilga A.V., Viola L., Quantum entanglement via nilpotent polynomials, Phys. Rev. A 74 (2006), 022331, 34 pages, quant-ph/0508234. 